\title{
Gauge theory on a space with linear Lie type fuzziness
}

\author{
Mohammad Khorrami 1 \\ Amir H. Fatollahi 2 \\ Ahmad Shariati 3 \\ Department of Physics, Alzahra University, Tehran 1993891167, Iran
}

\begin{abstract}
The U(1) gauge theory on a space with Lie type noncommutativity is constructed. The construction is based on the group of translation in Fourier space, which in contrast to space itself is commutative. In analogy with lattice gauge theory, the object playing the role of flux of field strength per plaquette, as well as the action, are constructed. It is observed that the theory, in comparison with ordinary $\mathrm{U}(1)$ gauge theory, has an extra gauge field component. This phenomena is reminiscent of similar ones in formulation of $\mathrm{SU}(N)$ gauge theory in space with canonical noncommutativity, and also appearance of gauge field component in discrete direction of Connes' construction of the Standard Model.
\end{abstract}

PACS: 11.15.-q, 02.40.Gh

Keyword: Gauge field theories, Noncommutative geometry

\footnotetext{
${ }^{1}$ mamwad@mailaps.org

${ }^{2}$ fath@alzahra.ac.ir

${ }^{3}$ shariati@mailaps.org
} 


\section{Introduction}

In recent years much attention has been paid to the formulation and study of field theories on noncommutative spaces. The motivation is partly the natural appearance of noncommutative spaces in some areas of physics, and the recent one in string theory. In particular it has been understood that the longitudinal directions of D-branes in the presence of a constant B-field background appear to be noncommutative, as seen by the ends of open strings 14.4. In this case the coordinates satisfy the canonical relation

$$
\left[\hat{x}_{\mu}, \hat{x}_{\nu}\right]=\mathrm{i} \theta_{\mu \nu} 1
$$

in which $\theta$ is an antisymmetric constant tensor and 1 is the unit operator. The theoretical and phenomenological implications of such noncommutative coordinates have been extensively studied during the last decade.

One direction to extend studies on noncommutative spaces is to consider spaces where the commutators of the coordinates are not constants. Examples of this kind are the noncommutative cylinder and the $q$-deformed plane (the Manin plane [5]), the so-called $\kappa$-Poincaré algebra [6] (see also [7-17]), and linear noncommutativity of the Lie algebra type [18] (see also 19 22]). In the latter the dimensionless spatial position operators satisfy the commutation relations of a Lie algebra:

$$
\left[\hat{x}_{a}, \hat{x}_{b}\right]=f_{a b}^{c} \hat{x}_{c},
$$

where $f_{a b}^{c}$ 's are structure constants of a Lie algebra. One example of this kind is the algebra $\mathrm{SO}(3)$, or $\mathrm{SU}(2)$. A special case of this is the so called fuzzy sphere 23] (see also 24 26]), where an irreducible representation of the position operators is used which makes the Casimir of the algebra, $\left(\hat{x}_{1}\right)^{2}+\left(\hat{x}_{2}\right)^{2}+\left(\hat{x}_{3}\right)^{2}$, a multiple of the identity operator (a constant, hence the name sphere). One can consider the square root of this Casimir as the radius of the fuzzy sphere. This is, however, a noncommutative version of a two-dimensional space (sphere).

In [27 29] a model was introduced in which the representation was not restricted to an irreducible one, instead the whole group was employed. In particular the regular representation of the group was considered, which contains all representations. As a consequence in such models one is dealing with the whole space, rather than a sub-space, like the case of fuzzy sphere as a 2-dimensional surface. In 27] basic ingredients for calculus on a linear fuzzy space, as well as basic notions for a field theory on such a space, were introduced. In [28, 29] basic elements for calculating the matrix elements corresponding to transition between initial and final states, together with the explicit expressions for tree and one-loop amplitudes were given. It is observed that models based on Lie algebra type noncommutativity enjoy three features:

- They are free from any ultraviolet divergences if the group is compact.

- There is no momentum conservation in such theories.

- In the transition amplitudes only the so-called planar graphs contribute. 
The reason for latter is that the non-planar graphs are proportional to $\delta$ distributions whose dimensions are less than their analogues coming from the planar sector, and so their contributions vanish in the infinite-volume limit usually taken in transition amplitudes [29. One consequence of different mass-shell condition of these kinds of theory was explored in [30.

In 31] the classical mechanics defined on a space with $\mathrm{SU}(2)$ fuzziness was studied. In particular, the Poisson structure induced by noncommutativity of $\mathrm{SU}(2)$ type was investigated, for either the Cartesian or Euler parameterization of SU(2) group. The consequences of SU(2)-symmetry in such spaces on integrability, was also studied in [31. In 32 the quantum mechanics on a space with SU(2) fuzziness was examined. In particular, the commutation relations of the position and momentum operators corresponding to spaces with Lie-algebra noncommutativity in the configuration space, as well as the eigen-value problem for the SU(2)-invariant systems were studied. The consequences of the Lie type noncommutativity of space on thermodynamical properties have been explored in 33 35.

The purpose of this work is to develop a gauge theory on a space with linear Lie type noncommutativity. In the same line as [27-29], the coordinates are taken to be in the regular representation of the Lie algebra. The gauge theory in space-time with canonical commutation relation (1) has been the subject of a large number of research attempts; see 1, 36 38 for a list of earlier attempts. One remarkable feature of the gauge theory on a canonical space is the absence of decoupling of the $\mathrm{U}(1)$ sector of $\mathrm{U}(N)$ from its $\mathrm{SU}(N)$ sector. This phenomenon can be explained on purely algebraic as well as string theoretic grounds [39. From the algebraic point of view, the reason can be traced back to the fact that the $\mathrm{SU}(N)$ sector is simply not closed under the $\star$-product between group elements, hence starting from pure $\mathrm{SU}(N)$ one always ends up with extended components for gauge fields, turning $\mathrm{SU}(N)$ to $\mathrm{U}(N)$. This phenomenon is mentioned here, as it will be seen that a similar phenomenon occurs here as well. It will be seen that the algebra used to construct the gauge field, requires an extra component for the gauge filed, here called $H$, even though one is considering $\mathrm{U}(1)$ symmetry. This observation is to some extent reminiscence of the appearance of gauge field component in the discrete direction of the two-sheet construction of the Standard Model by A. Connes, based on the Noncommutative Geometry approach 4042 . In that construction the gauge field component in the discrete direction between two sheets happens to be the Higgs field in the Standard Model point of view. As we will see, due to the lattice nature of space in our model [27 29], by analogy with Connes' construction, the appearance and the role of extra component for the gauge field is quite expected.

The scheme of the rest of this paper is the following. In section 2, a brief introduction of the group algebra is given, mainly to fix notation. In section 3 the gauge transformation on the so called matter field is introduced and a recipe is given to construct from a theory which is invariant under global gauge transformations, one that is invariant under local gauge transformation. The idea is essentially the same as minimal coupling applied to lattice theories. In 
section 4 , the connection on a commutative lattice is reformulated in such a way that can be generalized to the case of noncommutative spaces. In section 5 the connection in the noncommutative space is discussed. In section 6 the gauge invariant action corresponding to a (complex) matter field and a gauge field is presented. Section 7 is devoted to concluding remarks.

\section{The group algebra}

Assume that there exists a unique measure $\mathrm{d} U$ (up to a multiplicative constant) with the invariance properties

$$
\begin{aligned}
& \mathrm{d}(V U)=\mathrm{d} U, \\
& \mathrm{~d}(U V)=\mathrm{d} U, \\
& \mathrm{~d}\left(U^{-1}\right)=\mathrm{d} U,
\end{aligned}
$$

for any arbitrary element $(V)$ of the group. For a compact group $G$, such a measure does exist. There are, however, groups which are not compact but for them as well such a measure exists. Examples are noncompact Abelian groups.

The meaning of (3), is that the measure is invariant under the left-translation, right-translation, and inversion. This measure, the (left-right-invariant) Haar measure, is unique up to a normalization constant, which defines the volume of the group:

$$
\int_{G} \mathrm{~d} U=\operatorname{vol}(G)
$$

Using this measure, one constructs a vector space as follows. Corresponding to each group element $U$ an element $\mathfrak{e}(U)$ is introduced, and the elements of the vector space are linear combinations of these elements:

$$
f:=\int \mathrm{d} U f(U) \mathfrak{e}(U),
$$

The group algebra is this vector space, equipped with the multiplication

$$
f \bullet g:=\int \mathrm{d} U \mathrm{~d} V f(U) g(V) \mathfrak{e}(U V),
$$

where $(U V)$ is the usual product of the group elements. $f(U)$ and $g(U)$ belong to a field (here the field of complex numbers). It can be seen that if one takes the central extension of the group $\mathrm{U}(1) \times \cdots \times \mathrm{U}(1)$, the so-called Heisenberg group, with the algebra (1), the above definition results in the well-known star product of two functions, provided $f$ and $g$ are interpreted as the Fourier transforms of the functions.

So there is a correspondence between functionals defined on the group, and the group algebra. The definition (6) can be rewritten as

$$
(f \bullet g)(W)=\int \mathrm{d} V f\left(W V^{-1}\right) g(V) .
$$


The delta distribution is defined through

$$
\int \mathrm{d} U \delta(U) f(U):=f(\mathbf{1}),
$$

where $\mathbf{1}$ is the identity element of the group.

Next, one can define an inner product on the group algebra. Defining

$$
\begin{aligned}
\langle\mathfrak{e}(U), \mathfrak{e}(V)\rangle & :=\mathfrak{e}^{\dagger}(U) \mathfrak{e}(V), \\
& :=\delta\left(U^{-1} V\right),
\end{aligned}
$$

and demanding that the inner product be linear with respect to its second argument and antilinear with respect to its first argument, one arrives at

$$
\begin{aligned}
\langle f, g\rangle & =f^{\dagger} g, \\
& =\int \mathrm{d} U f^{*}(U) g(U),
\end{aligned}
$$

where $*$ is complex conjugation.

Finally, one defines a star operation through

$$
f^{\star}(U):=f^{*}\left(U^{-1}\right) .
$$

This is in fact equivalent to definition of the star operation in the group algebra as

$$
[\mathfrak{e}(U)]^{\star}:=\mathfrak{e}\left(U^{-1}\right) .
$$

It is then easy to see that

$$
\begin{aligned}
(f g)^{\star} & =g^{\star} f^{\star}, \\
\langle f, g\rangle & =\left(f^{\star} g\right)(\mathbf{1}) .
\end{aligned}
$$

One notes that if $f$ is a member of the group algebra, then $f^{\star}$ is a member of the group algebra as well, but $f^{\dagger}$ is a member of the space dual to the group algebra, that is, $f^{\dagger}$ acts linearly on the group algebra (as a vector space) and results in a scalar (a complex number).

\section{The gauge transformation}

The action for a complex scalar field $\phi$ in a noncommutative space can be written, similar to [27, as

$$
S_{0}=\int \mathrm{d} t\left\{\left(\dot{\phi}^{\star} \bullet \dot{\phi}\right)(\mathbf{1})+\left[\phi^{\star} \bullet(O \phi)\right](\mathbf{1})-\sum_{j=2}^{n} \frac{g_{j}}{2^{j} j !}\left[\left(\phi^{\star} \bullet \phi\right)^{j}\right](\mathbf{1})\right\}
$$

where

$$
(O \phi)(U)=O(U) \phi(U),
$$


and $O(U)$ is a scalar. One also has

$$
O\left(U^{-1}\right)=O^{*}(U)
$$

and that $g_{j}$ 's are real, to make sure that the action is real. It is seen that the combination $\left(\phi^{\star} \bullet \phi\right)$ is invariant under the gauge transformation

$$
\phi^{\prime}=f \bullet \phi,
$$

provided the function $f$ is unimodular:

$$
f^{\star} \bullet f=1 .
$$

To make the whole action gauge invariant, one way is to use the so called minimal coupling, changing the derivatives into covariant derivatives. Regarding the time derivative, one uses $\nabla_{0}$ instead of $\partial_{0}$ (the time derivative):

$$
\begin{aligned}
\nabla_{0} \phi & :=\partial_{0} \phi-\varphi \bullet \phi, \\
\nabla_{0} \phi^{\star} & :=\partial_{0} \phi^{\star}+\phi^{\star} \bullet \varphi,
\end{aligned}
$$

where $\varphi$ is pure imaginary:

$$
\varphi^{\star}=-\varphi
$$

and is gauge transformed like

$$
\varphi^{\prime}=f \bullet \varphi \bullet f^{-1}-f \bullet\left(\partial_{0} f^{-1}\right) .
$$

Derivatives with respect to the space coordinates are in $O$. The combination $\left[\phi^{\star} \bullet(O \phi)\right]$ is not invariant under the gauge transformation (18), as $\left(O \phi^{\prime}\right)$ is $[O(f \bullet \phi)]$ which is not equal to $[f \bullet(O \phi)]$. This is very much like the case of gauge theories on commutative spaces, where under gauge transformation (in which the field is multiplied by a group element valued function) the derivative of the field is not just multiplied by the same group element valued function. In the case of gauge theories on commutative spaces, one way to make the theory gauge invariant is to substitute differentiation with covariant differentiation, so that under gauge transformation the covariant derivative of the field does behave like the field itself, that is, the covariant derivative is multiplied by the same group element function. The covariant differentiation operator is then defined so that under gauge transformation it undergoes a similarity transformation, with the same group element valued function. Here a similar thing is done. The operator $O$ is replaced by an operator $\tilde{O}$, which is similarity transformed under the gauge transformation (18):

$$
\tilde{O}^{\prime} \phi=f \bullet \tilde{O}\left(f^{-1} \bullet \phi\right) .
$$

The operator $O$ contains derivatives with respect to the space coordinates. So its effect on the field would be to multiply its Fourier transformation by Fourier variables. As the Fourier variables corresponding to the space coordinates are group elements, the effect of $O$ on the field $\phi$ would be multiplying $\phi(U)$ by some 
function of $U$. A function of group can be written as a linear combination of matrix functions of the group (matrix elements of the irreducible representations of the group). Hence, defining $\mathrm{E}_{\lambda}$ through

$$
\left(\mathrm{L}_{\lambda} \phi\right)(U):=U_{\lambda} \phi(U),
$$

where $\lambda$ labels the representations, and $U_{\lambda}$ is the $\lambda$ representation of $U$, the action of $O$ would be

$$
\begin{aligned}
(O \phi)(U) & =\sum_{\lambda} \operatorname{tr}\left(a_{\lambda} U_{\lambda}\right) \phi(U), \\
& =: \sum_{\lambda} \operatorname{tr}\left[a_{\lambda}\left(\mathrm{L}_{\lambda} \phi\right)(U)\right],
\end{aligned}
$$

where $a_{\lambda}$ 's are constant matrices.

In the case of gauge theories on commutative spaces, the covariant derivative is constructed from the ordinary derivative by introducing another field (the gauge field) so that a combination of the ordinary derivative and the gauge field is similarity transformed under the gauge transformation. Here a similar thing is done. $\tilde{O}$ is defined based on $O$, using the gauge field $\mathcal{A}$, as

$$
\tilde{O} \phi:=\sum_{\lambda} \operatorname{tr}\left[a_{\lambda} \mathcal{A}_{\lambda} \bullet\left(\mathrm{L}_{\lambda} \phi\right)\right],
$$

and it would ensure (23) provided the operators $\mathcal{A}_{\lambda}$ are gauge transformed like

$$
\mathcal{A}_{\lambda}^{\prime} \bullet\left(\mathrm{L}_{\lambda} \phi\right)=f \bullet \mathcal{A}_{\lambda} \bullet\left[\mathrm{L}_{\lambda}\left(f^{-1} \bullet \phi\right)\right] .
$$

\section{The connection in the commutative space}

Consider a space $\mathbb{R}^{n}$. The (space component of the) connection is a vector with the components $A^{a}$, which are pure imaginary functions of space (and time), the gauge transformation of which under the action of a unimodular function $f$ is

$$
\mathrm{m}\left(A^{\prime a}\right)=\mathrm{m}(f) \mathrm{m}\left(A^{a}\right) \mathrm{m}\left(f^{-1}\right)+\mathrm{m}(f) \mathrm{m}\left(\partial^{a} f^{-1}\right),
$$

where $\mathrm{m}(f)$ is an operator which multiplies $f$ by the argument:

$$
[\mathrm{m}(f)] g:=f g .
$$

Note that the first term in the above is simply $\mathrm{m}\left(A^{a}\right)$, as the functions commute with each other. Alternatively, one can define a covariant derivative $\nabla$ by

$$
\nabla^{a}:=\partial^{a}+\mathrm{m}\left(A^{a}\right),
$$

and write the gauge transformation like

$$
\nabla^{\prime a}=\mathrm{m}(f) \nabla^{a} \mathrm{~m}\left(f^{-1}\right) .
$$


The (space-space component of the) curvature is an antisymmetric tensor $B$ of rank 2, related to $A$ through

$$
\mathrm{m}\left(B^{a b}\right)=\mathrm{m}\left(\partial^{a} A^{b}\right)-\mathrm{m}\left(\partial^{b} A^{a}\right)+\left[\mathrm{m}\left(A^{a}\right), \mathrm{m}\left(A^{b}\right)\right] .
$$

Again the third term vanishes as $A^{a}$ 's are commuting. There is another way to obtain the curvature, which can be generalized to the case of noncommutative spaces as well. One considers a set of generators $T_{a}$, which can be regarded as the generators of translation in the direction $a$ of the Fourier space, and build from the connection and the derivative the operators $\mathcal{A}_{\mathrm{s}}$ and $\mathrm{L}$ as

$$
\begin{aligned}
\mathcal{A}_{\mathrm{s}} & :=\exp \left(-\mathrm{i} \ell T_{a} A^{a}\right), \\
\mathrm{L} & :=\exp \left(-\mathrm{i} \ell T_{a} \partial^{a}\right),
\end{aligned}
$$

where $\ell$ is some parameter of the dimension length, and $A^{a}$ 's are pure imaginary functions. To be more rigorous, one should use $T_{a \lambda}$ instead of $T_{a}$, where $\lambda$ is a faithful representation of the Lie algebra corresponding to the group of translations on the Fourier space. So $\mathcal{A}_{\mathrm{s}}$ and $\mathrm{L}$ bear the subscript $\lambda$ as well. One further defines the operator $\mathrm{P}_{\mathrm{s}}$ as

$$
\mathrm{P}_{\mathrm{s}}:=\mathrm{m}\left(\mathcal{A}_{\mathrm{s}}\right) \mathrm{L} .
$$

It is seen that if

$$
\mathrm{P}_{\mathrm{s}}^{\prime}=\mathrm{m}(f) \mathrm{P}_{\mathrm{s}} \mathrm{m}\left(f^{-1}\right),
$$

then for small $\ell$, keeping only the first nonvanishing term in $\ell$ one arrives at (28). So one could begin from $\mathcal{A}_{\mathrm{s}}, \mathrm{L}$, and $\mathrm{P}_{\mathrm{s}}$, and define $\nabla$ as

$$
T_{a} \nabla^{a}:=\lim _{\ell \rightarrow 0}\left[(-\mathrm{i} \ell)^{-1}\left(\mathrm{P}_{\mathrm{s}}-1\right)\right] .
$$

Now define $\mathcal{B}_{\mathrm{s}}$ as

$$
\mathrm{m}\left(\mathcal{B}_{\mathrm{s}}\right):=\left(\mathrm{P}_{\mathrm{s}} \otimes \mathrm{P}_{\mathrm{s}}\right)\left(\mathrm{P}_{\mathrm{s}}^{-1} \otimes \mathrm{P}_{\mathrm{s}}^{-1}\right),
$$

in which the tensor product is defined only on the matrix structure coming from $T_{a}$ 's. As a consequence, even on ordinary (commutative) spaces, $\mathcal{B}_{\mathrm{s}}$ is not identity, as

$$
\begin{aligned}
(C \otimes D)^{\alpha \beta}{ }_{\gamma \delta} & =[(C \otimes 1)(1 \otimes D)]^{\alpha \beta}{ }_{\gamma \delta}, \\
& =C^{\alpha}{ }_{\gamma} D^{\beta}{ }_{\delta},
\end{aligned}
$$

while

$$
[(1 \otimes D)(C \otimes 1)]^{\alpha \beta}{ }_{\gamma \delta}=D^{\beta}{ }_{\delta} C^{\alpha}{ }_{\gamma} .
$$

So if the matrix elements of $C$ and $D$ do not commute with each other, [ $(1 \otimes$ $D)(C \otimes 1)]$ is not necessarily equal to $[(C \otimes 1)(1 \otimes D)]$, which is the same as $(C \otimes$ $D)$. This is the case, even if the space is commutative, as the matrix elements involved contain diferentiation as well as function multiplications, which do not necessarily commute. 
It is also seen from the Baker-Campbell-Hausdorff formula that the right hand side of (38) is actually the multiplication of something, as all of the commutators of derivatives and function multiplications are function multiplications.

One has

$$
\begin{aligned}
\lim _{\ell \rightarrow 0}\left\{(-\mathrm{i} \ell)^{-2}\left[\mathrm{~m}\left(\mathcal{B}_{\mathrm{s}}\right)-1\right]\right\}= & {\left[T_{a \mathrm{I}} \partial^{a}, T_{b \amalg} \mathrm{m}\left(A^{b}\right)\right]+\left[T_{a \mathrm{I}} \mathrm{m}\left(A^{a}\right), T_{b \amalg} \partial^{b}\right] } \\
& +\left[T_{a \mathrm{I}} \mathrm{m}\left(A^{a}\right), T_{b \amalg} \mathrm{m}\left(A^{b}\right)\right],
\end{aligned}
$$

where

$$
\begin{aligned}
T_{a \mathrm{I}} & :=T_{a} \otimes 1, \\
T_{a \text { II }} & :=1 \otimes T_{a} .
\end{aligned}
$$

So,

$$
\left(T_{a} \otimes T_{b}\right) \mathrm{m}\left(B^{a b}\right)=\lim _{\ell \rightarrow 0}\left\{(-\mathrm{i} \ell)^{-2}\left[\mathrm{~m}\left(\mathcal{B}_{\mathrm{s}}\right)-1\right]\right\},
$$

and again it is seen that the last term in (41) is vanishing.

Finally, defining

$$
\nabla_{0}:=\partial_{0}-\mathrm{m}(\varphi)
$$

where $\varphi$ is a function (the scalar potential), one can construct $E$ (the space-time component of the curvature) as follows. First define $\mathcal{E}_{\mathrm{s}}$ as

$$
\mathrm{m}\left(\mathcal{E}_{\mathrm{s}}\right):=\mathrm{P}_{\mathrm{s}} \nabla_{0} \mathrm{P}_{\mathrm{s}}^{-1}-\nabla_{0} .
$$

It is seen that

$$
\lim _{\ell \rightarrow 0}\left[(-\mathrm{i} \ell)^{-1} \mathrm{~m}\left(\mathcal{E}_{\mathrm{s}}\right)\right]=-T_{a} \mathrm{~m}\left(\partial^{a} \varphi+\partial_{0} A^{a}\right),
$$

so that if one defines

$$
E^{a}:=-\left(\partial^{a} \varphi+\partial_{0} A^{a}\right),
$$

then

$$
\lim _{\ell \rightarrow 0}\left[(-\mathrm{i} \ell)^{-1} \mathcal{E}_{\mathrm{s}}\right]=T_{a} \mathrm{~m}\left(E^{a}\right) .
$$

One can extend the definition of $\mathcal{A}_{\mathrm{s}}$ to $\mathcal{A}$, so that in the exponent there exists another term which is a multiple of the scalar matrix:

$$
\mathcal{A}:=\exp \left(-\mathrm{i} \ell T_{C} A^{C}\right),
$$

where capital letters as index can take the value $\mathrm{h}$ in addition to the values corresponding to the space directions. $T_{\mathrm{h}}$ is a pure imaginary multiple of the unit matrix, and $A^{\mathrm{h}}$ (denoted by $H$ ) is a pure imaginary function, being transformed as

$$
H^{\prime}=f H f^{-1} .
$$

$\mathcal{A}$ is a unitary function, but unlike $\mathcal{A}_{\mathrm{s}}$ is not necessarily unimodular. 
Extending the above definition to other quantities (removing the subscript s), one arrives at

$$
\begin{aligned}
\mathrm{P} & :=\mathrm{m}(\mathcal{A}) \mathrm{L} \\
\mathrm{m}(\mathcal{B}) & :=(\mathrm{P} \otimes \mathrm{P})\left(\mathrm{P}^{-1} \otimes \mathrm{P}^{-1}\right), \\
\mathrm{m}(\mathcal{E}) & :=\mathrm{P} \nabla_{0} \mathrm{P}^{-1}-\nabla_{0},
\end{aligned}
$$

so that,

$$
\begin{aligned}
\lim _{\ell \rightarrow 0}\left[(-\mathrm{i} \ell)^{-1}(\mathrm{P}-1)\right]= & T_{a} \nabla^{a}+T_{\mathrm{h}} \mathrm{m}(H), \\
\lim _{\ell \rightarrow 0}\left\{(-\mathrm{i} \ell)^{-2}[\mathrm{~m}(\mathcal{B})-1]\right\}= & \left(T_{a} \otimes T_{b}\right) \mathrm{m}\left(B^{a b}\right) \\
& +\left(T_{a} \otimes T_{\mathrm{h}}-T_{\mathrm{h}} \otimes T_{a}\right) \mathrm{m}\left(\partial^{a} H\right), \\
\lim _{\ell \rightarrow 0}\left[(-\mathrm{i} \ell)^{-1} \mathrm{~m}(\mathcal{E})\right]= & T_{a} \mathrm{~m}\left(E^{a}\right)-T_{\mathrm{h}} \mathrm{m}\left(\partial_{0} H\right) .
\end{aligned}
$$

Of course these can be written like

$$
\begin{aligned}
\lim _{\ell \rightarrow 0}\left[(-\mathrm{i} \ell)^{-1}(\mathrm{P}-1)\right] & =T_{C} \nabla^{C}, \\
\lim _{\ell \rightarrow 0}\left\{(-\mathrm{i} \ell)^{-2}[\mathrm{~m}(\mathcal{B})-1]\right\} & =\left(T_{C} \otimes T_{D}\right) \mathrm{m}\left(B^{C D}\right), \\
\lim _{\ell \rightarrow 0}\left[(-\mathrm{i} \ell)^{-1} \mathrm{~m}(\mathcal{E})\right] & =T_{C} \mathrm{~m}\left(E^{C}\right),
\end{aligned}
$$

where we have set

$$
\partial^{\mathrm{h}}:=0 .
$$

Using these, one can write the Lagrangian density for the gauge fields (and the field $H$ ) as

$$
\mathcal{L}_{\mathrm{G}}=\frac{\hbar}{2 g^{2}} \lim _{\ell \rightarrow 0}\left[\ell^{-4} \operatorname{tr}(\mathcal{B}-1)+\ell^{-2} \operatorname{tr}\left(\mathcal{E}^{2}\right)\right],
$$

where the generators $T_{C}$ have been taken so that

$$
\operatorname{tr}\left(T_{C} T_{D}\right)=-\delta_{C D},
$$

and $g$ is a dimensionless coupling constant. In the more explicit form,

$$
\mathcal{L}_{\mathrm{G}}=\frac{\hbar}{2 g^{2}}\left[\frac{1}{2} B^{a b} B_{a b}-E^{a} E_{a}+\left(\partial^{a} H\right)\left(\partial_{a} H\right)-\left(\partial_{0} H\right)^{2}\right] .
$$

The dimensions of the gauge fields $A$ and $H$ are (length) $)^{-1}$, those of $B$ and $E$ are (length $)^{-2}$, so the dimension of the Lagrangian density above is the dimension of action times (length) ${ }^{-4}$. The Lagrangian is the Lagrangian density integrated over space, or the value of the Fourier transformed Lagrangian density calculated at the origin. It is seen that in this Lagrangian density, the field $H$ does appear but it is not coupled to the other components of the gauge field. 
It is instructive to mention that the action (61), with the limit $\ell \rightarrow 0$ removed and also without the $H$ component, does in fact coincide with the continuous time version of the Wilson U(1) lattice gauge theory [43]. Of course in this case, the functions involved are periodic in momenta, and the integration region of the momenta is compact.

For the so called matter part of the Lagrangian density, one has

$$
\delta_{a b} \partial^{a} \partial^{b}=-\lim _{\ell \rightarrow 0}\left[(-\mathrm{i} \ell)^{-2} \operatorname{tr}\left(\mathrm{L}+\mathrm{L}^{-1}-2\right)\right] .
$$

Substituting $\mathrm{L}$ in the right-hand side with its covariant form $\mathrm{P}$, one arrives at

$$
\begin{aligned}
\delta_{C D} \nabla^{C} \nabla^{D} & =-\lim _{\ell \rightarrow 0}\left\{(-\mathrm{i} \ell)^{-2} \operatorname{tr}\left[\mathrm{m}(\mathcal{A}) \mathrm{L}+\mathrm{L}^{-1} \mathrm{~m}\left(\mathcal{A}^{-1}\right)-2\right]\right\}, \\
& =\delta_{a b} \nabla^{a} \nabla^{b}+\mathrm{m}\left(H^{2}\right) .
\end{aligned}
$$

So the matter part of the Lagrangian density would be

$$
\begin{aligned}
\mathcal{L}_{\mathrm{M}}= & \left(\dot{\phi}^{*}+\phi^{*} \varphi\right)(\dot{\phi}-\varphi \phi)+\phi^{*}\left(\delta_{a b} \nabla^{a} \nabla^{b}\right) \phi-\mu^{2} \phi^{*} \phi-\sum_{j=2}^{n} \frac{g_{j}}{2^{j} j !}\left(\phi^{*} \phi\right)^{j} \\
& +H^{2} \phi^{*} \phi .
\end{aligned}
$$

This is the usual gauge invariant Lagrangian density for a self interacting scalar field, with an additional term, the interaction of the field $H$ with the scalar field.

\section{The connection in the noncommutative space}

The (space component of the) connection in the representation $\lambda$ is a nonsingular function $\mathcal{A}_{\lambda}$ defined on the group with values in $\operatorname{GL}\left(\mathbb{V}_{\lambda}\right)$, where $\mathbb{V}_{\lambda}$ is the linear space on which the representation $\lambda$ acts. So,

$$
\mathcal{A}_{\lambda}=\int \mathrm{d} U \mathcal{A}_{\lambda}(U) \mathfrak{e}(U)
$$

where the integration runs over the group with a Haar measure as the integration measure, and $\mathfrak{e}(U)$ is that basis vector in the group algebra $\mathcal{G}$ corresponding to the group $G$, which corresponds to the element $U$ of the group $G$. By nonsingular it is meant that there exists a function $\mathcal{A}_{\lambda}^{-1}$ such that

$$
\begin{aligned}
\mathcal{A}_{\lambda} \bullet \mathcal{A}_{\lambda}^{-1} & =\mathcal{A}_{\lambda}^{-1} \bullet \mathcal{A}_{\lambda}, \\
& =\mathbf{1}_{\lambda} \mathfrak{e}(\mathbf{1}) .
\end{aligned}
$$

$\mathcal{A}$ is taken to be unitary:

$$
\mathcal{A}^{\dagger}=\mathcal{A}^{-1} \text {. }
$$

Another operator is $\mathrm{L}_{\lambda}$ (the left action in the representation $\lambda$ ) acting on $\mathbb{V}_{\lambda} \otimes \mathcal{G}:$

$$
\mathrm{L}_{\lambda}[v \mathfrak{e}(U)]:=\left(U_{\lambda} v\right) \mathfrak{e}(U) .
$$


To make the notation simpler, where no explicit use of the representation is made, the subscript $\lambda$ will be omitted.

Defining

$$
[\mathrm{m}(f)] g:=f \bullet g,
$$

for any two members $f$ and $g$ of the group algebra, and

$$
\mathcal{O}(U, V):=\mathfrak{e}^{\dagger}(U) \mathcal{O} \mathfrak{e}(V),
$$

for a linear operator $\mathcal{O}$ from $\mathcal{G}$ to $\mathcal{G}$, it is seen for an operator $\mathcal{O}$ there exists a member $g$ of $\mathcal{G}$ such that

$$
\mathcal{O}=\mathrm{m}(g),
$$

if and only if $\mathcal{O}(U, V)$ is a function of $\left(U V^{-1}\right)$, as

$$
\mathfrak{e}^{\dagger}(U) \mathrm{m}(g) \mathfrak{e}(V)=g\left(U V^{-1}\right) .
$$

An operator $\mathcal{O}$ is called a function multiplication, if there exists a $g$ in $\mathcal{G}$ such that (73) is satisfied. It is easy to see that a linear combination or product of two function multiplications are function multiplications. In fact

$$
\mathrm{m}\left(\alpha_{1} g_{1}+\alpha_{2} g_{2}\right)=\alpha_{1} \mathrm{~m}\left(g_{1}\right)+\alpha_{2} \mathrm{~m}\left(g_{2}\right),
$$

where $\alpha_{i}$ 's are constants, and

$$
\mathrm{m}\left(g_{1} \bullet g_{2}\right)=\mathrm{m}\left(g_{1}\right) \mathrm{m}\left(g_{2}\right) .
$$

Also if $g$ is nonsingular,

$$
[\mathrm{m}(g)]^{-1}=\mathrm{m}\left(g^{-1}\right) .
$$

The parallel transport operator $\mathrm{P}$ is defined as

$$
\mathrm{P}:=\mathrm{m}(\mathcal{A}) \mathrm{L} .
$$

It is seen that

$$
\mathrm{P}_{\lambda}(U, V)=\mathcal{A}_{\lambda}\left(U V^{-1}\right) V_{\lambda} .
$$

The gauge transformation of the parallel transport operator corresponding to a member $f$ of $\mathcal{G}$ is $\mathrm{P}^{\prime}$ defined as

$$
\mathrm{P}^{\prime}:=\mathrm{m}(f) \mathrm{P} \mathrm{m}\left(f^{-1}\right) .
$$

One has

$$
\begin{aligned}
\mathrm{P}^{\prime}(U, V)= & \int \mathrm{d} W \mathrm{~d} X f\left(U W^{-1}\right) \mathcal{A}\left(W X^{-1}\right) X f^{-1}\left(X V^{-1}\right), \\
= & \int \mathrm{d} W \mathrm{~d} X f\left[\left(U V^{-1}\right)\left(W V^{-1}\right)^{-1}\right] \\
& \times \mathcal{A}\left[\left(W V^{-1}\right)\left(X V^{-1}\right)^{-1}\right]\left(X V^{-1}\right) f^{-1}\left(X V^{-1}\right) V .
\end{aligned}
$$


This shows that there exists a function $\mathcal{A}^{\prime}$ such that

$$
\mathrm{P}^{\prime}=\mathrm{m}\left(\mathcal{A}^{\prime}\right) \mathrm{L} .
$$

So the gauge transformed connection $\mathcal{A}^{\prime}$ is defined as

$$
\mathcal{A}^{\prime}(U):=\int \mathrm{d} W \mathrm{~d} X f\left(U W^{-1}\right) \mathcal{A}\left(W X^{-1}\right) X f^{-1}(X) .
$$

One could attempt to amend (69) with another condition, something like unimodularity. Then, using a two dimensional representation of $\mathrm{SU}(2)$, one would be left with three (real) degrees of freedom for the (space part of) the gauge field. The above gauge transformation, however, does not preserve such a condition. So even for a unimodular representation, one should include gauge fields corresponding to nonunimodular matrices. That's the reason the field $H$ was introduced in the previous section.

To define the (space-space component of the) curvature, we prove that there exists a function $\mathcal{B}_{\lambda}$ such that

$$
\left(\mathrm{P}_{\lambda} \otimes \mathrm{P}_{\lambda}\right)\left(\mathrm{P}_{\lambda}^{-1} \otimes \mathrm{P}_{\lambda}^{-1}\right)=\mathrm{m}\left(\mathcal{B}_{\lambda}\right) .
$$

Denoting the left hand side of (84) by $\mathrm{H}$, one has

$$
\begin{aligned}
& \mathrm{H}(U, V)=\int \mathrm{d} W \mathrm{~d} X \mathrm{~d} Y\left\{\left[\mathcal{A}_{\lambda}\left(U W^{-1}\right) W_{\lambda}\right] \otimes\left[\mathcal{A}_{\lambda}\left(W X^{-1}\right) X_{\lambda}\right]\right\} \\
& \times\left\{\left[X_{\lambda}^{-1} \mathcal{A}_{\lambda}^{-1}\left(X Y^{-1}\right)\right] \otimes\left[Y_{\lambda}^{-1} \mathcal{A}_{\lambda}^{-1}\left(Y V^{-1}\right)\right]\right\} \\
&= \int \mathrm{d} W \mathrm{~d} X \mathrm{~d} Y\left[\mathcal{A}_{\lambda}\left(U W^{-1}\right)\left(W X^{-1}\right)_{\lambda} \mathcal{A}_{\lambda}^{-1}\left(X Y^{-1}\right)\right] \\
& \otimes\left[\mathcal{A}_{\lambda}\left(W X^{-1}\right)\left(X Y^{-1}\right)_{\lambda} \mathcal{A}_{\lambda}^{-1}\left(Y V^{-1}\right)\right]
\end{aligned}
$$

showing that the left hand side depends on only $\left(U V^{-1}\right)$. So $\mathcal{B}_{\lambda}$ according to (84) is well defined. It is easily seen that this curvature undergoes a similarity transformation under the gauge transformation:

$$
\mathcal{B}_{\lambda}^{\prime}=f \bullet \mathcal{B}_{\lambda} \bullet f^{-1},
$$

where $\mathcal{B}^{\prime}$ is the gauge transformed curvature corresponding to a gauge transformation with the function $f$.

The time component of the connection is denoted be $\varphi \cdot \varphi$ is a member of $\mathcal{G}$, using which $\nabla_{0}$ (the covariant time derivative) is defined as

$$
\nabla_{0}:=\partial_{0}-\mathrm{m}(\varphi)
$$

$\varphi^{\prime}$ (the gauge transformed $\varphi$ ) is defined such that the action of gauge transformation on $\nabla_{0}$ be a similarity transformation with $\mathrm{m}(f)$ :

$$
\nabla_{0}^{\prime}=\mathrm{m}(f) \nabla_{0} \mathrm{~m}\left(f^{-1}\right),
$$


from which one arrives at

$$
\varphi^{\prime}=f \bullet \varphi \bullet f^{-1}-f \bullet\left[\partial_{0}\left(f^{-1}\right)\right] .
$$

Finally, the space-time component of the curvature in the representation $\lambda$ is denoted by $\mathcal{E}_{\lambda}$, defined through

$$
\mathrm{m}\left(\mathcal{E}_{\lambda}\right):=\mathrm{P}_{\lambda} \nabla_{0} \mathrm{P}_{\lambda}^{-1}-\nabla_{0} .
$$

It has to be shown that the $\mathrm{m}(\mathcal{E})$ from (90) is well defined, that is, the right hand side of (90) is a function multiplication. Noting that $\partial_{0}$ commutes with $\mathrm{L}$, it is seen that

$$
\mathrm{P}_{\lambda} \nabla_{0} \mathrm{P}_{\lambda}^{-1}-\nabla_{0}=\mathrm{m}\left\{\mathcal{A}_{\lambda} \bullet\left[\partial_{0}\left(\mathcal{A}_{\lambda}^{-1}\right)\right]\right\}-\mathrm{P}_{\lambda} \mathrm{m}(\varphi) \mathrm{P}_{\lambda}^{-1}+\mathrm{m}(\varphi) .
$$

One also has

$$
\mathfrak{e}^{\dagger}(U) \mathrm{L}_{\lambda} \mathrm{m}(\varphi) \mathrm{L}_{\lambda}^{-1} \mathfrak{e}(V)=\left(U V^{-1}\right)_{\lambda} \varphi\left(U V^{-1}\right),
$$

showing that $\mathrm{L}_{\lambda} \mathrm{m}(\varphi) \mathrm{L}_{\lambda}^{-1}$ is a function multiplication, from which it turns out that the right hand side of (90) is a function multiplication. Using (90), and the transformation properties of $\mathrm{P}$ and $\nabla_{0}$, it is seen that the action of gauge transformation on $\mathcal{E}$ is a similarity transformation with $f$ :

$$
\mathcal{E}_{\lambda}^{\prime}=f \bullet \mathcal{E}_{\lambda} \bullet f^{-1} .
$$

\section{The action}

The gauge action is defined similar to the case of commutative space, the Lagrangian density for which was defined in (61). First, one notes that if the function $X$ is similarity transformed under a gauge transformation:

$$
X^{\prime}=f \bullet X \bullet f^{-1},
$$

then the value of that function at identity is gauge invariant:

$$
\begin{aligned}
X^{\prime}(\mathbf{1}) & =\int \mathrm{d} U \mathrm{~d} V f\left(U^{-1}\right) X\left(U V^{-1}\right) f^{-1}(V), \\
& =\int \mathrm{d} U^{\prime} \mathrm{d} V f\left(V^{-1} U^{\prime-1}\right) X\left(U^{\prime}\right) f^{-1}(V), \\
& =\int \mathrm{d} U^{\prime} \delta\left(U^{\prime}\right) X\left(U^{\prime}\right), \\
& =X(\mathbf{1}) .
\end{aligned}
$$

Based on this, the pure gauge field sector of the Lagrangian is defined as

$$
L_{\mathrm{G}}=\frac{\hbar}{2 g^{2}}\left[\ell^{-4} \operatorname{tr}(\mathcal{B}-1)+\ell^{-2} \operatorname{tr}\left(\mathcal{E}^{2}\right)\right](\mathbf{1}) .
$$


Using

$$
\begin{array}{r}
\mathcal{B}(\mathbf{1})=\int \mathrm{d} U \mathrm{~d} V \mathrm{~d} W\left[\mathcal{A}\left(U V^{-1}\right) V W^{-1} \mathcal{A}^{-1}(W)\right] \\
\otimes\left[\mathcal{A}\left(U^{-1}\right) U V^{-1} \mathcal{A}^{-1}\left(V W^{-1}\right)\right],
\end{array}
$$

and

$$
\begin{aligned}
\mathcal{E}(U)= & \int \mathrm{d} V \mathcal{A}\left(U V^{-1}\right)\left(\partial^{0} \mathcal{A}^{-1}\right)(V) \\
& +\varphi(U)-\int \mathrm{d} V \mathrm{~d} W \mathcal{A}\left(\left(U V^{-1}\right) V W^{-1} \varphi\left(V W^{-1}\right) \mathcal{A}^{-1}(W),\right.
\end{aligned}
$$

one can write the gauge Lagrangian more explicitly as

$$
\begin{aligned}
L_{\mathrm{G}}=\frac{\hbar}{2 g^{2} \ell^{4}} & \left\{\int \mathrm{d} U \mathrm{~d} V \mathrm{~d} W \operatorname{tr}\left[\mathcal{A}\left(U V^{-1}\right) V W^{-1} \mathcal{A}^{-1}(W)\right]\right. \\
\times & \left.\operatorname{tr}\left[\mathcal{A}\left(U^{-1}\right) U V^{-1} \mathcal{A}^{-1}\left(V W^{-1}\right)\right]-[\operatorname{tr}(1)]^{2} \delta(\mathbf{1})\right\} \\
& +\frac{\hbar}{2 g^{2} \ell^{2}} \int \mathrm{d} U \operatorname{tr}\left[\mathcal{E}(U) \mathcal{E}\left(U^{-1}\right)\right] .
\end{aligned}
$$

It may look that the above Lagrangian is singular, due to the singular term $\delta(\mathbf{1})$. It is not. One way to see this, is to write everything in terms of $(\mathcal{A}-1)$ instead of $\mathcal{A}$, so that the term proportional to $\delta(1)$ is canceled. Alternatively, one may use the logarithm of $\mathcal{A}$ as the gauge field, to show manifestly that the above Lagrangian is not singular.

The matter part of the Lagrangian corresponding to (15) and (66) is

$$
\begin{aligned}
L_{\mathrm{M}}= & {\left[\left(\dot{\phi}^{\star}+\phi^{\star} \bullet \varphi\right) \bullet(\dot{\phi}-\varphi \bullet \phi)\right](\mathbf{1})+\ell^{-2}\left\{\phi^{\star} \bullet\left[\operatorname{tr}\left(\mathcal{A} \mathrm{L}+\mathrm{L}^{-1} \mathcal{A}-2\right) \phi\right]\right\}(\mathbf{1}) } \\
& -\mu^{2}\left(\phi^{\star} \bullet \phi\right)(\mathbf{1})-\sum_{j=2}^{n} \frac{g_{j}}{2^{j} j !}\left[\left(\phi^{\star} \bullet \phi\right)^{j}\right](\mathbf{1}) .
\end{aligned}
$$

\section{Concluding remarks}

An alternative construction of gauge theories on commutative spaces was discussed, which could be generalized to the case of Lie type noncommutative spaces. This alternative is basically constructed in terms of parallel transports. Based on that, a gauge theory on a noncommutative space of Lie type noncommutativity was constructed, in which the gauge field is basically some representation of the group the matrix elements of which are functions. Using this, and the time component of the gauge field, analogues of the electric and magnetic fields were constructed, from which a gauge action was built. Apart from differences proportional to the noncommutativity parameter, it is seen that one has to introduce an extra degree of freedom in the gauge field. The reason is that even for the simplest representation of the group $\mathrm{SU}(2)$, starting from a 
three component (space) gauge field corresponding to the two dimensional representation of three generators of the $\mathrm{SU}(2)$, applying the gauge transformation introduces a fourth component corresponding to a generator which commutes with the other three. In other words, there is no analogue of unimodularity of two dimensional matrices (for gauge fields) which is preserved under gauge transformation.

Acknowledgement: This work was supported by the research council of the Alzahra University. 


\section{References}

[1] N. Seiberg \& E. Witten, JHEP 9909 (1999) 032.

[2] A. Connes, M. R. Douglas, \& A. Schwarz, JHEP 9802 (1998) 003.

[3] M. R. Douglas \& C. Hull, JHEP 9802 (1998) 008.

[4] H. Arfaei \& M. M. Sheikh-Jabbari, Nucl. Phys. B526 (1998) 278.

[5] Yu. I. Manin, "Topics in Noncommutative Geometry", Princeton University Press, (1991).

[6] J. Lukierski, H. Ruegg, A. Nowicki \& V. N. Tolstoy, Phys. Lett. B264 (1991) 331.

[7] M. Chaichian, A. Demichev, \& P. Presnajder, Nucl. Phys. B567 (2000) 360 .

[8] M. Chaichian, A. Demichev, \& P. Presnajder, J. Math. Phys. 41 (2000) 1647.

[9] S. Majid \& H. Ruegg, Phys. Lett. B334 (1994) 348.

[10] J. Lukierski, H. Ruegg, \& W. J. Zakrzewski, Annals Phys. 243 (1995) 90.

[11] J. Lukierski \& H. Ruegg, Phys. Lett. B329 (1994) 189.

[12] G. Amelino-Camelia, Phys. Lett. B392 (1997) 283.

[13] G. Amelino-Camelia \& M. Arzano, Phys. Rev. D65 (2002) 084044.

[14] G. Amelino-Camelia, M. Arzano, \& L. Doplicher, in "25th Johns Hopkins Workshop on Current Problems in Particle Theory", hep-th/0205047.

[15] P. Kosinski, J. Lukierski, \& P. Maslanka, Phys. Rev. D62 (2000) 025004.

[16] D. Robbins \& S. Sethi, JHEP 07 (2003) 034.

[17] H. Grosse \& M. Wohlgenannt, Nucl. Phys. B748 (2006) 473.

[18] H. S. Snyder, Phys. Rev. 71 (1947) 38.

[19] J. Madore, S. Schraml, P. Schupp, \& J. Wess, Eur. Phys. J. C16 (2000) 161.

[20] N. Sasakura, JHEP 0005 (2000) 015.

[21] S. Imai \& N. Sasakura, JHEP 0009 (2000) 032.

[22] Y. Sasai \& N. Sasakura, 0711.3059 [hep-th].

[23] J. Madore, Class. Quant. Grav. 9 (1992) 69. 
[24] P. Presnajder, Mod. Phys. Lett. A18 (2003) 2431.

[25] H. Grosse \& P. Presnajder, Lett. Math. Phys. 46 (1998) 61.

[26] H. Grosse \& P. Presnajder, Lett. Math. Phys. 33 (1995) 171.

[27] A. H. Fatollahi \& M. Khorrami, Europhys. Lett. 80 (2007) 20003.

[28] H. Komaie-Moghaddam, A. H. Fatollahi, \& M. Khorrami, Eur. Phys. J. C53 (2008) 679 .

[29] H. Komaie-Moghaddam, M. Khorrami, \& A. H. Fatollahi, Phys. Lett. B661 (2008) 226.

[30] A. Shariati, M. Khorrami, \& A. H. Fatollahi, Europhys. Lett. 81 (2008) 40003.

[31] M. Khorrami, A. H. Fatollahi, \& A. Shariati, J. Math. Phys. 50 (2009) 072902 .

[32] A. H. Fatollahi, A. Shariati, \& M. Khorrami, Eur. Phys. J. C60 (2009) 489.

[33] H. Shin \& K. Yoshida, Nucl. Phys. B701 (2004) 380.

[34] W.-H. Huang, JHEP 0908 (2009) 102.

[35] A. Shariati, M. Khorrami, \& A. H. Fatollahi, J. Phys. A: Math. Theor. 43 (2010) 285001.

[36] M. R. Douglas \& N. A. Nekrasov, Rev. Mod. Phys. 73 (2001) 977.

[37] R. J. Szabo, Phys. Rept. 378 (2003) 207.

[38] A. Konechny \& A. Schwarz, Phys. Rept. 360 (2002) 353.

[39] L. Bonora, M. Schnabl, M.M. Sheikh-Jabbari \& A. Tomasiello, Nucl. Phys. B 589 (2000) 461-474.

[40] A. Connes, Noncommutative Geometry (Academic Press, New York 1994).

[41] A. Connes \& J. Lott, Nucl. Phys. Proc. Supp. B 18 (1990) 295.

[42] D. Kastler, Rev. Math. Phys. 5 (1993) 477.

[43] K. G. Wilson, Phys. Rev. D 10 (1974) 2445. 